\title{
Simulación de la Producción de Energía Eléctrica con Aerogeneradores de Pequeño Tamaño
}

\author{
Alex M. Araújo*, Gilberto M. Melo, Armando L. R. de Medeiros y Maurílio J. dos Santos \\ Universidade Federal de Pernambuco, Departamento de Engenharia Mecânica, Programa de \\ Pós-Graduação em Engenharia Mecânica, Av. Acadêmico Hélio Ramos 6000, Cidade Universitária, \\ 50740-530 Recife, PE-Brasil (e-mail: ama@ufpe.br)
}

*autor a quien debe ser dirigida la correspondencia

\begin{abstract}
Resumen
Este trabajo se presenta la simulación de la producción de electricidad a partir de varios aerogeneradores de pequeño tamaño, en el régimen de vientos de Olinda-Pernambuco-Brasil, teniendo en cuenta una serie de registros de velocidades del viento y la temperatura en 1998. Los datos de velocidad de viento fueron discretizados a intervalos apropiados para permitir una mejor manipulación de la información contenida en las curvas de energía eléctrica, suministradas por los fabricantes. La herramienta permite realizar un análisis comparativo del rendimiento de aerogeneradores, tanto en el aspecto de la producción de energía generada de cada aerogenerador como de los rendimientos en función de la velocidad del viento. Los resultados muestran que el sistema de los vientos locales influyó en la producción de electricidad y que los datos facilitados por los fabricantes deben ser certificados.
\end{abstract}

Palabras clave: energía eólica, aerogeneradores, electricidad, régimen de vientos

\section{Simulation of the Production of Electricity with Small Size Aerogenerators}

\begin{abstract}
This paper presents a simulation of electricity production from several small wind turbines in the wind regime of Olinda-PE-Brazil, taking into account a number of records wind speeds and temperature in 1998, with the aim of developing a performance analysis tool that allows its selection. The hourly wind speed data were discretized at appropriate intervals to allow better handling of the information contained in the curves of electricity, supplied by manufacturers. The tool allows a comparative analysis of the performance of wind turbines, both in the aspect of the electrical energy production as its efficiency in function of wind speed. The results show that of the local wind regime influenced the production of electricity and that the data provided by manufacturers must be certified.
\end{abstract}

Keywords: wind energy, wind turbines, electricity, wind regime 


\section{INTRODUCCIÓN}

La Agencia Nacional de Energía Eléctrica (ANEEL, 2002) clasifica como aerogeneradores de pequeño tamaño a máquinas eólicas generadoras con potencia nominal igual o inferior a $500 \mathrm{~kW}$. La International Electrotechnical Commission (IEC, 2006) considera de pequeño tamaño a aquellas con área barrida entre 2 y $200 \mathrm{~m}^{2}$.

La selección y aplicación de aerogeneradores de pequeño tamaño para la generación de energía eléctrica es una alternativa tecnológica muy poco utilizada aun en Brasil. A través de un análisis comparativo entre el coste del kWh generado por un aerogenerador de pequeño tamaño con uno de gran tamaño, se puede constatar que los mercados potenciales que van a ser ocupados por ellos son distintos, o sea, mientras la energía generada por la de gran tamaño sería direccionada a la venta de las concesionarias, aquella generada por las de pequeño tamaño actuaría en el segmento de auto-producción, de acuerdo con Hopkins (1999) y Hirahara et al. (2005).

De acuerdo con la información técnica de la concesionaria de energía del estado de Pernambuco (PE) en Brasil, la composición del valor final de la venta de la energía indica que el sector de generación contribuye con aproximadamente $35 \%$ del coste, faltando transmisión, distribución, impuestos específicos del sector eléctrico y otros tributos para completar el valor total. Esa diferencia unida a una investigación rigurosa para la identificación del local adecuado para la instalación de la turbina de pequeño tamaño, existencia de líneas de financiación de largo plazo, mejora de la tecnología con reducción de los costes de los equipamientos y reglamentación del sector eléctrico, deberán conducir a una mayor difusión en el empleo de los aerogeneradores de pequeño tamaño tanto aislados como conectados directamente en la red eléctrica.

Un análisis de la evaluación de la viabilidad técnica de la aplicación de aerogeneradores de pequeño tamaño ha sido desarrollado por Celik (2003) y utiliza los parámetros de forma y escala de Weibull de la distribución de viento real. Otro análisis, con base en las curvas de distribución de energía eléctrica de los aerogeneradores con un viento real, se presentó en Pallabazzer (2004) para calcular la electricidad producida. Actualmente, esta búsqueda de herramientas tecnológicas está en desarrollo en diversas partes del mundo, de acuerdo con Bishop y Amaratunga (2008) y Peacock et al. (2008).

No hay procedimientos técnicos específicos en la legislación brasileña para guiar la estimación de la energía generada por un aerogenerador a un régimen de viento. En este artículo se propuso un procedimiento metodológico basado en una variante de la metodología de intervalos, que se presentan en IEC61400-12 (1998), utilizado por Castro (2007), sobre la base de datos horarios de velocidad de viento discretizados en rangos de $0,5 \mathrm{~m} / \mathrm{s}$.

\section{METODOLOGIA}

\section{Datos eólicos}

Los datos de viento y temperatura del aire utilizado en este trabajo fueron medidos por la UFPE Universidad Federal de Pernambuco (Centro Brasileño de Energía Eólica), en el área de pruebas de los aerogeneradores, localizada en la ciudad costera de Olinda-PE, durante el período comprendido entre diciembre de 1996 y diciembre de 1998. Por razones de pérdidas menores de datos colectados, se eligió el año de 1998 para ese estudio, así mismo en esa serie histórica ocurrieron pérdidas de registros referentes a los períodos del 06 al 17 de abril (273 muestras) y del 19 de septiembre al 03 de octubre (337 muestras), lo que representó cerca de 6,9\% del total de los datos. Los datos faltantes fueron completados con base en la matriz de transición de Markov aplicado en el software Windographer, que mantiene las propiedades estadísticas de la serie.

Las mediciones fueron realizadas a una altura de $20 \mathrm{~m}$ sobre el nivel del suelo con una tasa de muestra de $1 \mathrm{~Hz}$ y registradas a cada diez minutos. Los datos fueron procesados, vía integración, para obtener las medias horarias. Fueron utilizados en las mediciones anemómetros, termómetros y loggers, con las especificaciones indicadas en la Tabla $1 .$. 
Tabla 1: Equipos utilizados en las mediciones

\begin{tabular}{|c|c|c|c|}
\hline Equipo & Tipo del sensor & Rango del sensor & Incertidumbre \\
\hline $\begin{array}{c}\text { Anemómetro } \\
\text { Maximum \#40 }\end{array}$ & Anemómetro de 3 cazoletas & $1 \mathrm{~m} / \mathrm{s}$ a $96 \mathrm{~m} / \mathrm{s}$ & $\pm 0.1 \mathrm{~m} / \mathrm{s}$ \\
\hline Termómetro & $\begin{array}{c}\text { Circuito integrado; sensor de temperatura con } \\
\text { seis placas; escudo de radiación }\end{array}$ & $-40{ }^{\circ} \mathrm{C}$ a $52.5^{\circ} \mathrm{C}$ & $\pm 1.11^{\circ} \mathrm{C}$ \\
\hline
\end{tabular}

Las principales características estadísticas de la serie usada fueron estimadas con la ayuda de modelos simplificados (Mathew, 2006). Los resultados encontrados fueron: velocidad media anual $(5,6 \mathrm{~m} / \mathrm{s})$, desviación estándar $(1,8 \mathrm{~m} / \mathrm{s})$, y constantes de escala y de forma de la distribución de Weibull $(k=3,49)$ y $(c=6,29)$, respectivamente. La Figura $1(a)$ muestra la evolucion de las medias mensuales de la velocidad del viento y la Figura 1(b) muestra la distribución de la media horaria a lo largo del día, relativas al año de 1998.
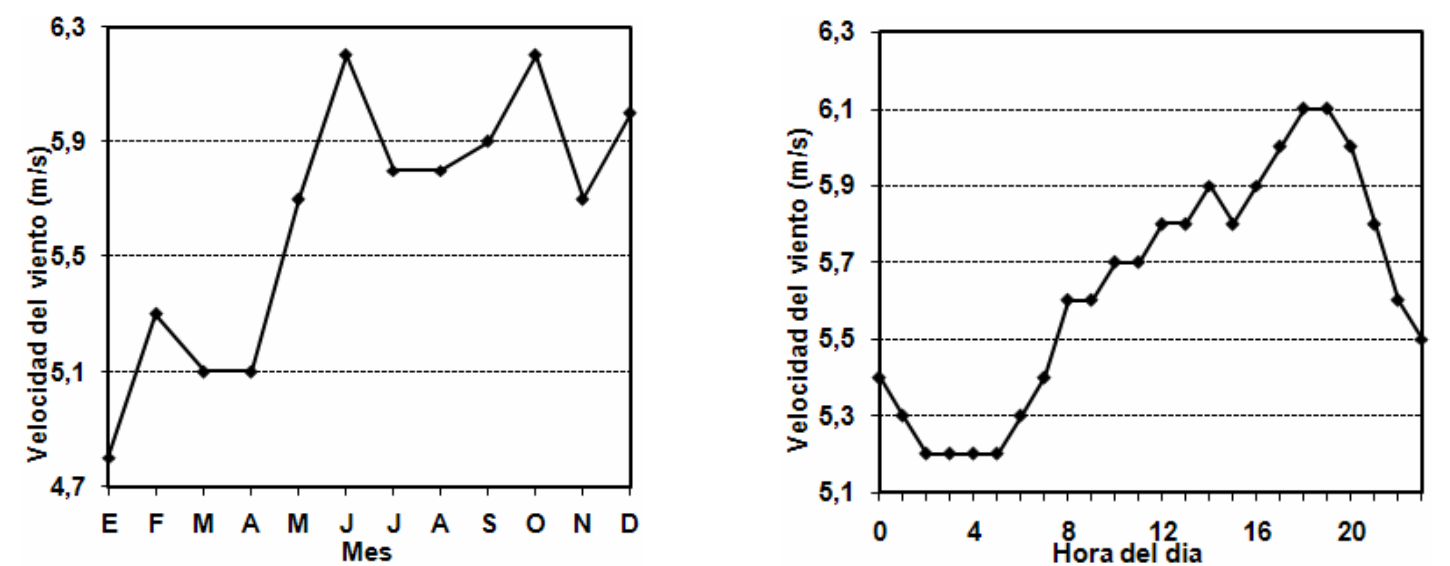

Fig. 1: (a) Media mensual de las velocidades de viento y (b) Perfil diario de la velocidad del viento.

\section{Procedimiento}

Fue realizado un estudio de la distribución de las frecuencias de las velocidades del viento en rangos de $0,5 \mathrm{~m} / \mathrm{s}$, de acuerdo con el método de intervalos descrito en IEC61400-12 (1998), variando esta segmentación desde 0 a 12,5 m/s (amplitud de las variaciones de las medias horarias de los datos trabajados), siendo el primer rango entre 0 y $0,75 \mathrm{~m} / \mathrm{s}$. En este caso, la serie representante de valores de velocidad del viento $(\mathrm{u})$, resultante de la división en rangos, es: $0,5-1,0-1,5-\ldots-12,0-12,5$ $\mathrm{m} / \mathrm{s}$. Se utilizaron recursos del software Excel y se obtuvieron los agrupamientos de los datos de presentados en la Figura 2 que muestra el número de eventos, $F(n)$, contenidos en cada rango, versus la orden del rango, $\mathrm{n}$.

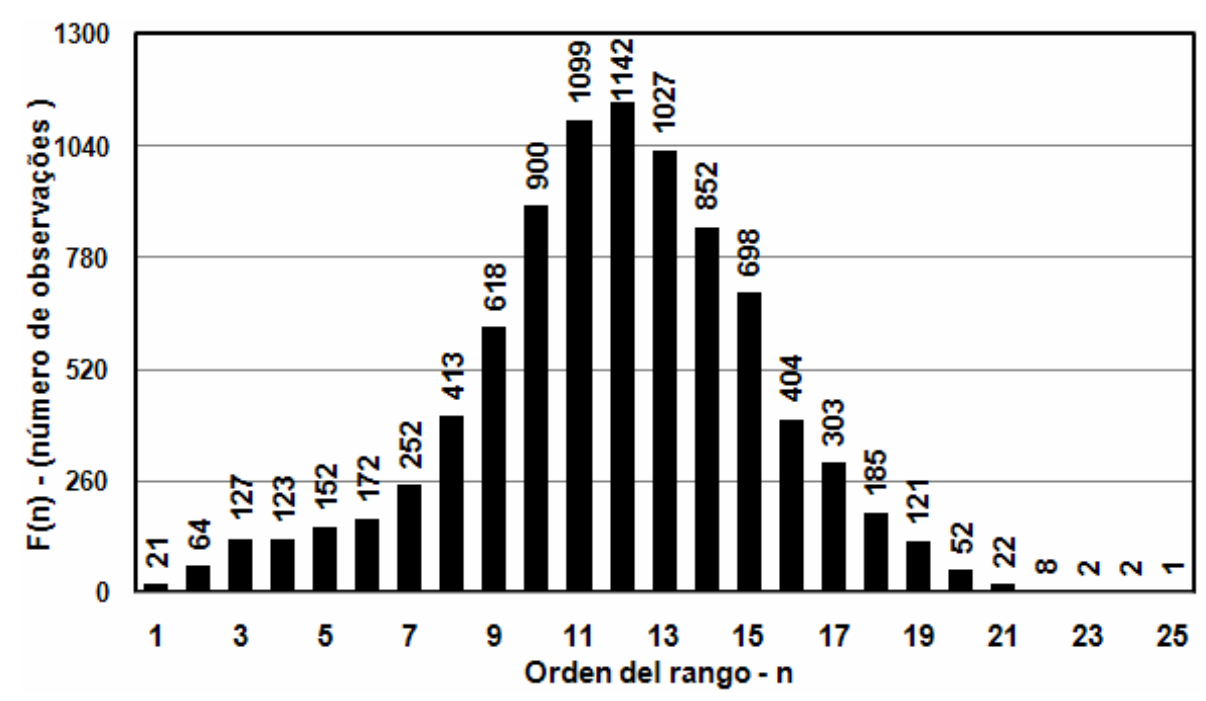

Fig. 2: Histograma de las velocidades del viento del año de 1998 
En la ecuación (1), los valores de $\mathrm{N}_{0}$ y $\mathrm{N}_{\text {máx }}$ corresponden a los límites de la gama de velocidades del viento en que el sistema produce energía, es decir, entre la velocidad de arranque $u_{0}[\mathrm{~m} / \mathrm{s}$ ] y la velocidad de abatimiento $u_{\text {máx }}[\mathrm{m} / \mathrm{s}]$ (Castro, 2007).

$\mathrm{E}_{\mathrm{a}}=\sum_{\mathrm{n}=\mathrm{N}_{0}}^{\mathrm{N}_{\max }} \mathrm{F}(\mathrm{n}) \cdot \mathrm{P}_{\mathrm{e}}(\mathrm{n})$

De esa forma, la energía anual producida por el aerogenerador eléctrico $\left(E_{a}[W h]\right)$ sería estimada por el producto de su potencia de salida $\left(\mathrm{P}_{\mathrm{e}}[\mathrm{W}]\right)$ por el número de horas encontrado en cada rango de velocidades de vientos, correspondiente al valor de $\mathrm{F}(\mathrm{n})$. En este artículo, se consideró un índice de operación del sistema de 100\%, según la IEC61400-12 (1998).

Las curvas de potencia eléctrica producida, suministradas por los fabricantes de aerogeneradores de pequeño tamaño fueron dibujadas en Autocad y se obtuvo los valores de $\mathrm{P}_{\mathrm{e}}(\mathrm{u})$ para las velocidades de referencia (u). A partir de esa información, se viabiliza el cálculo de la energía producida para cada rango de velocidades, de acuerdo con la ecuación (1).

En el cálculo de la energía producida anualmente, se consideró que el aerogenerador se instalaría a la altura de $20 \mathrm{~m}$, que es la altura correspondiente donde fueron medidos los datos de velocidad del viento y temperatura. Sin embargo, según IEC61400-12 (1998), es necesario verificar si el cambio de la densidad del aire durante el año estaria fuera de los márgenes que se recomienda para la densidad $1,225 \pm 0,050 \mathrm{~kg} / \mathrm{m}^{3}$. El proceso utilizado en el artículo, debido a los datos disponibles, fue el cálculo de la media anual de la densidad del aire $(\rho[\mathrm{kg} / \mathrm{m}])$ con una entrada de datos de las temperaturas medias por hora $(T[K])$ y altura $(Z[\mathrm{~m}])$ del anemómetro en el lugar de instalación del equipo, mediante la ecuación (2) (Mathew, 2006).

$\rho_{a}=\frac{353,049}{T} \cdot e^{\left(-0,034 \frac{Z}{T}\right)}$

\section{Rendimiento de los aerogeneradores}

Para la verificación de la consistencia teórica de las curvas de potencia eléctrica suministradas por los fabricantes y, consecuentemente, del resultado de las energías obtenidas con el método utilizado, se estimó que era necesario calcular los rendimientos de los aerogeneradores para cada valor representativo de los rangos de segmentación adoptadas para las velocidades eólicas. La estimación del rendimiento, $\eta$, para cada rango de velocidades de viento se consigue a través de la relación entre las potencias de salida $\left(P_{e}[k W]\right)$ del aerogenerador y la potencia teóricamente disponible $\left(P_{\text {disp }}[k W]\right)$ a las velocidades correspondientes, de acuerdo con la ecuación (3).

$\eta(u)=\frac{P_{e}(u)}{P_{\operatorname{disp}}(u)}$

En la ecuación (3) la potencia disponible es obtenida a través de la estimación del flujo de energía cinética pasando por el área del disco cubierto por el rotor $\left(A\left[\mathrm{~m}^{2}\right]\right)$, para cada rango de velocidades del viento de acuerdo con la ecuación (4).

$P \operatorname{disp}(u)=\frac{1}{2} \rho_{a} \cdot u^{3} A$

Donde $\rho_{\mathrm{a}}$ es la densidad del aire $\left(\mathrm{kg} / \mathrm{m}^{3}\right)$.

\section{RESULTADOS Y DISCUSIÓN}

En este artículo fue pre-seleccionado 5 aerogeneradores de pequeño tamaño con las características técnicas de diseño similar y la potencia nominal, tal y como se muestra en la Tabla 2. Se puede ver 
que los aerogeneradores tienen potencias nominales que van desde 5,0 a 6,0 kW, con velocidades nominales de viento de 12,0 a $14,0 \mathrm{~m} / \mathrm{s}$.

Tabla 2: Características técnicas de los aerogeneradores pre-seleccionados

\begin{tabular}{|c|c|c|c|c|c|c|}
\hline Fabricante & $\begin{array}{c}\text { Nombre } \\
\text { Comercial }\end{array}$ & $\begin{array}{c}\text { Potencia } \\
\text { Nominal } \\
(\mathrm{kW})\end{array}$ & $\begin{array}{c}\text { Velocidad de } \\
\text { Arranque } \\
(\mathrm{m} / \mathrm{s})\end{array}$ & $\begin{array}{c}\text { Velocidad } \\
\text { Nominal } \\
(\mathrm{m} / \mathrm{s})\end{array}$ & $\begin{array}{c}\mathrm{N}^{\circ} \text { de } \\
\text { Palas }\end{array}$ & $\begin{array}{c}\text { Diámetro } \\
(\mathrm{m})\end{array}$ \\
\hline Vergnet & GEV 6/5 & 5,0 & 3,5 & 14,0 & 2 & 6,0 \\
\hline Vergnet & GEV 5/5 & 5,0 & 3,0 & 14,0 & 2 & 5,0 \\
\hline Westwind & $5,5 \mathrm{KW}$ & 5,5 & 3,0 & 14,0 & 3 & 5,1 \\
\hline Eoltec & Scirocco & 6,0 & 2,7 & 11,5 & 2 & 5,6 \\
\hline J. B. Bornay & Inclin 6000 & 6,0 & 3,5 & 12,0 & 3 & 4,0 \\
\hline
\end{tabular}

Las curvas de potencia eléctrica dibujadas con ayuda del Autocad y las curvas de rendimiento obtenidas de acuerdo con el procedimiento propuesto en este artículo son mostradas en las Figuras 3(a) e 3(b), respectivamente.
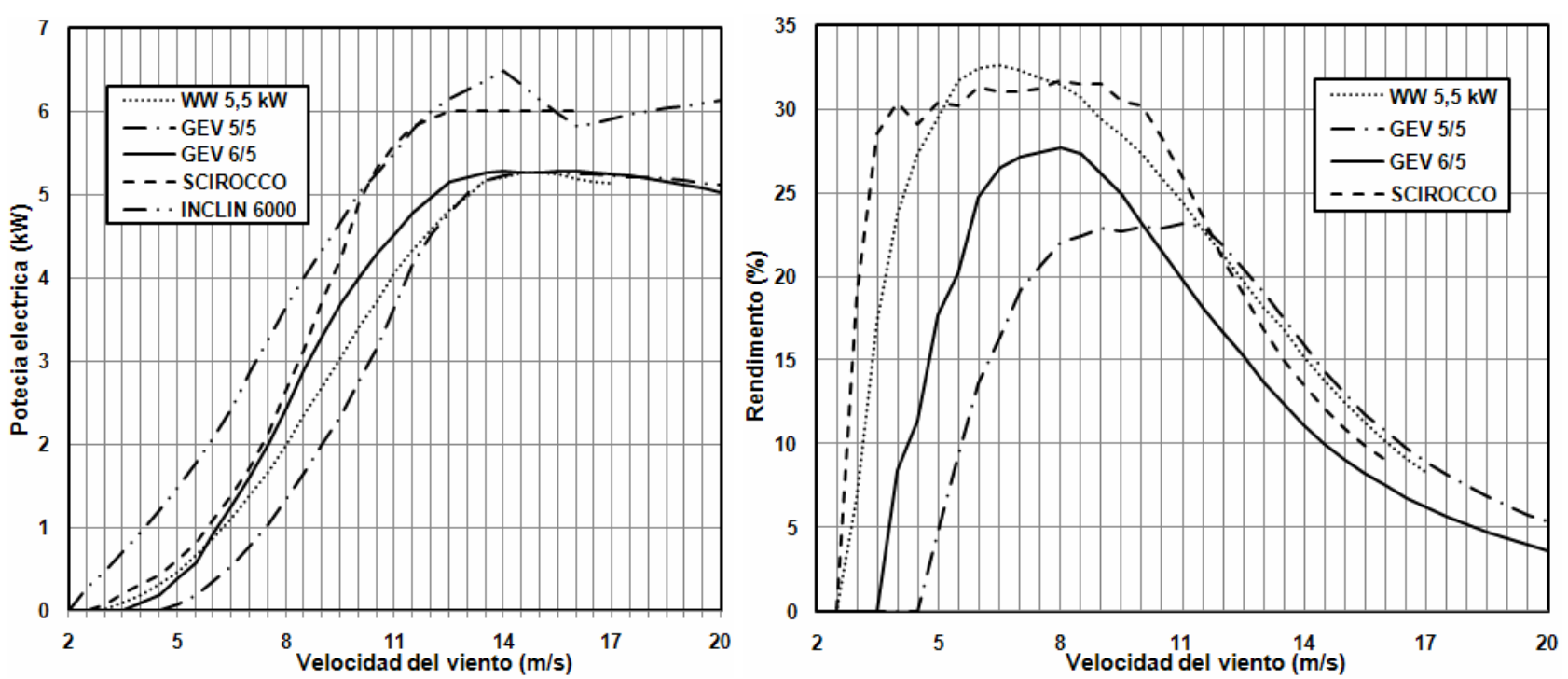

Fig. 3: (a) Curvas de potencia eléctrica y (b) Rendimiento, en función de la velocidad del viento.

La validación del procedimiento de obtención de las curvas de potencia eléctrica en forma gráfica de base única fue desarrollada para el aerogenerador de Eoltec, el único que suministró sus datos en forma gráfica y en tabla de valores. La comparación directa entre los valores de energía producida usando los dos tipos de información mostró una diferencia de aproximadamente $4 \%$. Este resultado indica que el procedimiento gráfico con base en Autocad apresenta pequeños errores.

Al calcular la electricidad producida, se examinó el impacto del cambio en la densidad del aire con la temperatura a una altura de $22 \mathrm{~m}$, lo que resulta en el valor promedio anual $\rho=1,172 \mathrm{~kg} / \mathrm{m}^{3}$. Durante el período considerado se registraron temperaturas que van desde 21,4 a $32,1^{\circ} \mathrm{C}$, con el valor medio de $27,5^{\circ} \mathrm{C}$ y una desviación estándar de $1,6{ }^{\circ} \mathrm{C}$. Estos resultados indican que no hay necesidad de hacer correcciones en el cálculo de la energía eléctrica producida por causa de variaciones en la densidad del aire, por lo tanto, su valor medio anual es casi el límite del rango recomendado por la IEC (1998) y la desviación estándar de la temperatura del aire es baja.

Los resultados obtenidos para la energía eléctrica producida, $\mathrm{E}_{\mathrm{a}}$, de acuerdo con la metodologia propuesta son presentados en la Tabla 3, que muestra tambien la producción de energía por $\mathrm{m}^{2}$, $\mathrm{E}_{a} / \mathrm{A}$.

La velocidad $u_{\text {máx }}$ no influenció el cálculo de la energía producida pues las velocidades máximas de Olinda-PE fueron menores que 12,5 m/s, todavía, umáx es mayor que $20 \mathrm{~m} / \mathrm{s}$ (Ackermann, 2008). 
Tabla 3: Producción de energía eléctrica de los aerogeneradores

\begin{tabular}{|l|l|c|c|}
\hline Fabricante & Nombre Comercial & $\mathrm{E}_{\mathrm{a}}(\mathrm{kWh})$ & $\mathrm{E}_{\mathrm{a}} / \mathrm{A}\left(\mathrm{kWh} / \mathrm{m}^{2}\right)$ \\
\hline Vergnet & GEV 6/5 & 9.618 & 340,2 \\
\hline Vergnet & GEV 5/5 & 4.596 & 254,1 \\
\hline Westwind & $5,5 \mathrm{KW}$ & 8.651 & 423,5 \\
\hline Eoltec & Scirocco & 11.193 & 423,6 \\
\hline J. B. Bornay & Inclin 6000 & 18.814 & $1.497,2$ \\
\hline
\end{tabular}

La diferencia encontrada en la energía anual producida entre los dos aerogeneradores del fabricante Vergnet, puede explicarse a partir de las Figuras 3(a) y 3(b). En la Figura 3(a), la energía eléctrica generada por los dos aerogeneradores son similares sólo para velocidades de los vientos fuertes, por encima de $10 \mathrm{~m} / \mathrm{s}$. Por otro lado, en la Figura 2, el número de observaciones de la velocidad del viento superior o igual a $10 \mathrm{~m} / \mathrm{s}$ es una parcela de aproximadamente $1 \%$ del total. Por esta razón, la producción de energía es muy poco influenciada por los vientos de esta magnitud. En la Figura 3(a), el rango de velocidades de viento que se va de 4 a $8 \mathrm{~m} / \mathrm{s}$ representa $82 \%$ del total de los datos registrados. En este rango de velocidades, la energía eléctrica generada por el aerogenerador GEV $6 / 5$ es superior a la GEV 5/5, como puede verse en la Figura 3(a). Esto explica una producción de energía a partir del aerogenerador GEV 6/5 de 109\% mayor en relación al GEV 5/5, para el régimen de vientos de Olinda-PE, en 1998. Teniendo en cuenta que los dos aerogeneradores tienen la misma potencia nominal, se puede decir que el aerogenerador GEV 6/5 sería mejor aplicado al caso.

La comparación de los aerogeneradores Vergnet con los demás presenta una amplia gama de resultados aparentemente incompatible con las características técnicas de cada aerogenerador. En este caso, el análisis de los resultados podría ser más preciso a través de la observación del rendimiento de cada aerogenerador, calculado por la ecuación (3) y presentado en la Figura 3(b), con la excepción del aerogenerador Inclin 6000 , que presentó rendimientos por encima del $100 \%$, incompatible con la teoría. La Tabla 4 presenta los valores de rendimientos para el rango de velocidades del viento de operación del aerogenerador Inclin 6000.

Tabla 4: Valores del rendimiento del aerogenerador Inclin 6000

\begin{tabular}{|l|c|c|c|c|c|c|c|c|c|c|}
\hline $\mathrm{u}(\mathrm{m} / \mathrm{s})$ & 3 & 4 & 5 & 6 & 7 & 8 & 9 & 10 & 11 & 12 \\
\hline$\eta(\%)$ & 229 & 193 & 155 & 125 & 109 & 93 & 77 & 65 & 54 & 45 \\
\hline
\end{tabular}

Este resultado obtenido para la máquina Inclin 6000 indica una posibilidad de error en la información de las especificaciones técnicas suministradas por el fabricante.

El valor del rendimiento, obtenido por la ecuación (3), considera tanto el valor del coeficiente de potencia como tambien las pérdidas de origén mecánica y eléctrica. Para aerogeneradores, la cantidad máxima para el coeficiente $\mathrm{Cp}$ se obtienen alrededor de 0,40 , aunque el límite teórico de $\mathrm{Cp}$ es 0,59, dado por el coeficiente de Betz (Lysen, 1983). Por esta razón, se puede decir que los rendimientos, en la Figura 3(b), son consistentes en todos los rangos de velocidad.

De la Tabla 3 se observa que los aerogeneradores Scirocco y Westwind son los que más producirían energia eléctrica por área barrida en el régimen de vientos de Olinda. Este resultado podría también ser explicado por la verificación en el gráfico de la Fig. 4 en que estos aerogeneradores presentan valores de alto rendimiento. Además el generador Scirocco presenta valores de Cp casi constante en una amplia gama de velocidades de viento. Este patrón de funcionamiento es típico en un aerogenerador de eje horizontal ideal. Este comportamiento no se observa en la curva de rendimiento del aerogenerador Westwind.

Los fabricantes de aerogeneradores eólicos de pequeño tamaño que suelen facilitar en catálogos sus datos técnicos y las curvas de las potencias eléctricas, mostrando dichos datos en forma gráfica. Tal procedimiento conduce a la necesidad de la discretización de la curva dada, incurriendo, con el procedimiento usado en este trabajo, en errores intrínsecos a ese proceso. El suministro por parte de los fabricantes de sus curvas de potencia eléctrica en modo analítico, variando con la velocidad de los vientos, a intervalos de $0,5 \mathrm{~m} / \mathrm{s}$, por ejemplo, minimizaría aquel error. 
Los datos proporcionados por los fabricantes solo permiten la evaluación del rendimiento del aerogenerador para cada velocidad de viento. Este valor incluye tanto el desempeño aerodinámico del rotor como las pérdidas en el proceso de conversión mecánico-eléctrica. Seria adecuado el suministro también de la curva del coeficiente de potencia que traería al proceso de selección una visión más amplia sobre la adecuación de su equipo al régimen de vientos de operación, y también un indicativo más claro del avance tecnológico presente en su proyecto en los aspectos aerodinámico y de conversión mecánico-eléctrica.

Ha sido posible constatar que para dos equipos fabricados por una misma empresa (GEV 5/5 e GEV $6 / 5$ ) y con la misma potencia nominal, el aumento del diámetro del rotor de $5 \mathrm{~m}$ para $6 \mathrm{~m}$ asociado a las diferencias de proyecto ha llevado a un aumento de $109 \%$ en la generación de energía para el régimen de viento de Olinda-PE en 1998. Dado que el aumento de área barrida por el rotor de GEV $6 / 5$ es de $44 \%$, podemos concluir que el proyecto de este aerogenerador es más apropiado para las condiciones de vientos en estudio, que explica la ganancia adicional del $45 \%$. En la Figura 4, la curva de rendimiento de la GEV $6 / 5$ presenta una velocidad inferior a la de inicio de la GEV 5/5, lo que permitiría al aerogenerador GEV 6/5 operar con mayor eficiencia en el rango de velocidades que prevalecen en Olinda-PE.

\section{CONCLUSIONES}

Los datos de los aerogeneradores estudiados presentan la velocidad nominal de $12 \mathrm{~m} / \mathrm{s}$ hasta $14 \mathrm{~m} / \mathrm{s}$ lo que representaría una aplicación inadecuada para las condiciones de vientos de Olinda-PE. La Figura 2 muestra que la ocurrencia de vientos mayores que $10 \mathrm{~m} / \mathrm{s}$ es inferior a $1 \%$ del total de observaciones. Este hecho causa un bajo valor del factor de la planta, alrededor del $24 \%$, que se define como el cociente entre la energía suministrada por el aerogenerador y la energía que sería generada con potencia nominal constante. Para aumentar este factor de la planta, sería necesario que los aerogeneradores analizados sean diseñados con velocidades nominales más bajas ya que el sistema de vientos locales presenta un factor de forma de Weibull alto $(k=3,49)$, típica del régimen de vientos constantes.

El procedimiento propuesto en este trabajo sugiere que tanto la velocidad media anual como el patrón de régimen de viento sean considerados como parámetros para definir las características técnicas que serán incorporadas en el no proyecto de aerogeneradores. En la etapa de preselección de los aerogeneradores, este mismo procedimiento deberá ser utilizado (Melo y Araújo, 2007).

Los resultados obtenidos con la simulación de la utilización de la turbina Inclin 6000 , indica y alerta para la necesidad de la certificación de las curvas de potencia eléctrica e de las curvas de coeficiente de potencia de los aerogeneradores por instituciones acreditadas por el mercado.

\section{AGRADECIMIENTOS}

Al "Conselho Nacional de Desenvolvimento Científico e Tecnológico (CNPq)" por el apoyo financiero. Al Profesor Jorge R. Henriquez y a los alumnos de maestría Breno A. Loureiro y Daniel A. A. Valença por la ayuda técnica.

\section{REFERENCIAS}

Ackermann, Thomas; Wind Power in Power Systems. 1st edition, pp. 25-51, John Wiley \& Sons Ltd, England (2008).

ANEEL-Agencia Nacional de Energia Eléctrica; Atlas de Energia Elétrica do Brasil (2002), http://www.aneel.gov.br/aplicacoes/atlas/pdf/06-Energia_Eolica(3).pdf. Acceso: 26 de Mayo (2007).

Bishop, J.D.K. y G.A.J. Amaratunga, Evaluation of small wind turbines in distributed arrangement as sustainable wind energy option for Barbados, Energy Conversion and Management: 49, 1652-1661 (2008).

Castro, Rui M.G.; Introdução a Energia Eólica. $3^{a}$ edição, pp. 50-58, IST, Lisboa, Portugal (2007). 
Celik, A.N.; Energy output estimation for small-scale wind power generators using Weibullrepresentative wind data, Renewable Energy: 91, 693-707 (2003).

Hirahara, H., M.Z. Hossain, M. Kawahashi e Y. Nonomura; Testing basic performance of a very small wind turbine designed for multi-purposes, Reneawble Energy: 30, 1279-1297(2005).

Hopkins, W.; Small to medium size wind turbines: Local of use a local resource, Renewable Energy: 16, 944-947(1999).

IEC 61400-2, Wind turbines - Design requirements for small wind turbines, Geneva, Switzerland (2006).

IEC 61400-12, Wind turbine power performance testing, 1-50, Geneva, Switzerland (1998).

Lysen, E.H.; Introduction do Wind Energy, $2^{\text {a }}$ edición, pp. 77-85, SWD Publications, Netherlands (1983).

Mathew, S.; Wind Energy - Fundamentals, Resource Analysis and Economics, pp. 61-88, Springer (2006).

Melo, G.M. y A.M. Araújo; Simulação da Produção de Energia Elétrica com Turbinas Eólicas de Pequeno Porte em Regime de Ventos de Olinda / BR. Actas del $8^{\circ}$ Congreso Iberoamericano de Ingeniería Mecánica, CD-ROM, Cusco-Perú, 23 a 25 de Octubre (2007).

Pallabazer, R.; Previsional estimation of the energy output of windgenerators, Renewable Energy: 29, 413-420 (2004).

Peacock, A.D. y otros cuatro autores; Micro wind turbines in the UK domestic sector, Energy and Buildings: 40 , 1324-1333 (2008). 